\title{
Characterization of nonhomogeneous dual wavelet frames in Sobolev spaces
}

\author{
Jian-Ping Zhang*, Qiang-Qiang Chang \\ College of Mathematics and Computer Science, Yan'an University, Shaanxi 716000 China \\ *Corresponding author, e-mail: zhjp198254@163.com
}

Received 15 Jun 2021

Accepted 25 Oct 2021

\begin{abstract}
In recent years, wavelets and wavelet frames have been extensively investigated by many researchers due to their great design freedom and the potential applications in many fields. In this work, we address nonhomogeneous wavelet frames in Sobolev spaces setting, a characterization of nonhomogeneous dual wavelet frames in $\left(H^{s}\left(\mathbb{R}^{d}\right), H^{-s}\left(\mathbb{R}^{d}\right)\right)$ is obtained, which generalizes the results of Proposition 2.3 in [Appl Comput Harmon Anal 36 (2014):51-62].
\end{abstract}

KEYWORDS: Bessel sequence, wavelet frame, nonhomogeneous dual wavelet frame, Sobolev space

MSC2010: 42C40 42C15

\section{INTRODUCTION}

In view of the great design freedom and the potential applications in image restoration, signal processing and many other fields, wavelets and wavelet frames have been extensively investigated by many researchers (see [1-3] for details). In particular, the homogeneous dual wavelet frames (also called affine dual frames) in $L^{2}\left(\mathbb{R}^{d}\right)$ were originally characterized by Han [4], and were later characterized by Bownik [5], some of their variations can be found in [68]. Mixed extension principles (MEP) provide us with an important method to construct homogeneous dual wavelet frames from refinable functions, which were first proposed by Ron and Shen $[9,10]$. Recently, Han and Shen $[11,12]$ generalized the MEP from $L^{2}\left(\mathbb{R}^{d}\right)$ to Sobolev spaces pairs $\left(H^{s}\left(\mathbb{R}^{d}\right), H^{-s}\left(\mathbb{R}^{d}\right)\right)$ for homogeneous dual wavelet frames construction, the smoothness and vanishing moment requirements of two systems were separated completely from each other. Consequently, the construction of dual wavelet frames in Sobolev spaces gets much easier. Nonhomogeneous wavelet frames have natural connections with homogeneous ones; for this type of wavelet frames, Han [13-15] and Romero et al [16] extensively studied them in both theory and application. Atreas et al [17] obtained a characterization of nonhomogeneous dual wavelet frames in $L^{2}\left(\mathbb{R}^{d}\right)$. Motivated by the above works, we obtain a characterization of nonhomogeneous dual wavelet frames in $\left(H^{s}\left(\mathbb{R}^{d}\right), H^{-s}\left(\mathbb{R}^{d}\right)\right)$, which generalizes the results of Proposition 2.3 in [17].

We begin with some notations. We use $\mathbb{Z}$ and $\mathbb{N}$ to represent the set of integers and the set of positive integers, respectively, and denote $\mathbb{N}_{0}=\mathbb{N} \cup$ $\{0\}$. Let $d \in \mathbb{N}$, we use $\mathbb{T}^{d}=[0,1)^{d}$ to represent a $d$-dimensional unit torus. Given a set $E$ on $\mathbb{R}^{d}$, let $|E|$ represent the Lebesgue measure of $E$, and let $\chi_{E}$ represent the characteristic function on $E$. For a function $f \in L^{1}\left(\mathbb{R}^{d}\right) \cap L^{2}\left(\mathbb{R}^{d}\right)$, its Fourier transform $\hat{f}$ is defined by

$$
\hat{f}(\cdot)=\int_{\mathbb{R}^{d}} f(x) \mathrm{e}^{-2 \pi i\langle x \cdot\rangle} \mathrm{d} x
$$

and is naturally extended to $L^{2}\left(\mathbb{R}^{d}\right)$, where $\langle\cdot, \cdot\rangle$ is the usual inner product on $\mathbb{R}^{d}$. Throughout this paper, we write $\delta$ as the Dirac sequence such that $\delta_{0,0}=1, \delta_{0, k}=$ 0 for $0 \neq k \in \mathbb{Z}^{d}$.

Given $s \in \mathbb{R}$, the Sobolev space $H^{s}\left(\mathbb{R}^{d}\right)$ consists of all distributions $f$ such that

$$
\|f\|_{H^{s}\left(\mathbb{R}^{d}\right)}^{2}=\int_{\mathbb{R}^{d}}|\hat{f}(\xi)|^{2}\left(1+\|\xi\|^{2}\right)^{s} \mathrm{~d} \xi<\infty
$$

where $\|\cdot\|$ is the usual norm on $\mathbb{R}^{d}$. Notice that $H^{s}\left(\mathbb{R}^{d}\right)$ is a separable Hilbert space under the following definition of inner product:

$$
\begin{array}{r}
\langle f, g\rangle_{H^{s}\left(\mathbb{R}^{d}\right)}=\int_{\mathbb{R}^{d}} \hat{f}(\xi) \overline{\hat{g}(\xi)}\left(1+\|\xi\|^{2}\right)^{s} \mathrm{~d} \xi, \\
\quad f, g \in H^{s}\left(\mathbb{R}^{d}\right) .
\end{array}
$$

Furthermore, for every $g \in H^{-s}\left(\mathbb{R}^{d}\right)$,

$$
\langle f, g\rangle=\int_{\mathbb{R}^{d}} \hat{f}(\xi) \overline{\hat{g}(\xi)} \mathrm{d} \xi, \quad f \in H^{s}\left(\mathbb{R}^{d}\right)
$$

gives a linear continuous functional in $H^{s}\left(\mathbb{R}^{d}\right)$. The Sobolev space $H^{-s}\left(\mathbb{R}^{d}\right)$ is the dual space of $H^{s}\left(\mathbb{R}^{d}\right)$, and vice versa.

We use $M^{*}$ to represent the conjugate transpose for a matrix $M$. A $d \times d$ matrix $M$ is said to be expansive if the moduli of all its eigenvalues are greater than 1 . In many literatures of wavelet frame theory, $M$ is required 
to be an expansive integer matrix including $M=2 I_{d}$, where $I_{d}$ is the identity matrix of order $d$. Let $M$ be a $d \times d$ expansive matrix. A function $\tau: \mathbb{Z}^{d} \rightarrow \mathbb{N}_{0}$ is defined by

$$
\tau(n)=\sup \left\{j \geqslant 0: M^{*^{-j}} n \in \mathbb{Z}^{d}\right\},
$$

and set $\tau(0)=\infty$. Moreover, for convenient narration, we always write

$$
m=|\operatorname{det} M|^{1 / d},
$$

and write

$$
f_{j, k}(\cdot)=m^{j d / 2} f\left(\left(M^{j} \cdot\right)-k\right)
$$

and

$$
\begin{aligned}
f_{j, k}^{s}(\cdot) & =m^{-j s} f_{j, k}(\cdot) \\
& =m^{j\left(\frac{d}{2}-s\right)} f\left(\left(M^{j} \cdot\right)-k\right), \quad j \in \mathbb{Z}, k \in \mathbb{Z}^{d}
\end{aligned}
$$

for a tempered distribution $f$.

Given $L \in \mathbb{N}$, let $\phi, \varphi_{1}, \varphi_{2}, \ldots, \varphi_{L} \in H^{s}\left(\mathbb{R}^{d}\right)$ and $\tilde{\phi}, \tilde{\varphi}_{1}, \tilde{\varphi}_{2}, \ldots, \tilde{\varphi}_{L} \in H^{-s}\left(\mathbb{R}^{d}\right)$, we denote by $X^{s}\left(\phi ; \varphi_{1}, \varphi_{2}, \ldots, \varphi_{L}\right)$ and $X^{-s}\left(\tilde{\phi} ; \tilde{\varphi}_{1}, \tilde{\varphi}_{2}, \ldots, \tilde{\varphi}_{L}\right)$ the following two nonhomogeneous wavelet systems in $H^{s}\left(\mathbb{R}^{d}\right)$ and $H^{-s}\left(\mathbb{R}^{d}\right)$, respectively.

$$
\begin{aligned}
X^{s}\left(\phi ; \varphi_{1}, \varphi_{2}, \ldots, \varphi_{L}\right)=\left\{\phi_{0, k}: k \in \mathbb{Z}^{d}\right\} \\
\cup\left\{\varphi_{l, j, k}^{s}: j \in \mathbb{N}_{0}, k \in \mathbb{Z}^{d}, l=1,2, \ldots, L\right\}
\end{aligned}
$$

and

$$
\begin{aligned}
X^{-s}\left(\tilde{\phi} ; \tilde{\varphi}_{1}, \tilde{\varphi}_{2}, \ldots, \tilde{\varphi}_{L}\right)=\left\{\tilde{\phi}_{0, k}: k \in \mathbb{Z}^{d}\right\} \\
\quad \cup\left\{\tilde{\varphi}_{l, j, k}^{-s}: j \in \mathbb{N}_{0}, k \in \mathbb{Z}^{d}, l=1,2, \ldots, L\right\} .
\end{aligned}
$$

We say that $X^{s}\left(\phi ; \varphi_{1}, \varphi_{2}, \ldots, \varphi_{L}\right)$ is a nonhomogeneous wavelet frame in $H^{s}\left(\mathbb{R}^{d}\right)$ if there exist two positive constants $A$ and $B$ such that

$$
\begin{aligned}
& A\|f\|_{H^{s}\left(\mathbb{R}^{d}\right)}^{2} \leqslant \sum_{k \in \mathbb{Z}^{d}}\left|\left\langle f, \phi_{0, k}\right\rangle_{H^{s}\left(\mathbb{R}^{d}\right)}\right|^{2} \\
&+\sum_{l=1}^{L} \sum_{j=0}^{\infty} \sum_{k \in \mathbb{Z}^{d}}\left|\left\langle f, \varphi_{l, j, k}^{s}\right\rangle_{H^{s}\left(\mathbb{R}^{d}\right)}\right|^{2} \\
& \leqslant B\|f\|_{H^{s}\left(\mathbb{R}^{d}\right)}^{2}, \quad \forall f \in H^{s}\left(\mathbb{R}^{d}\right),
\end{aligned}
$$

where $A$ and $B$ are called frame bounds. In particular, if $A=B(A=B=1)$ in $(11)$, then $X^{s}\left(\phi ; \varphi_{1}, \varphi_{2}, \ldots, \varphi_{L}\right)$ is said to be a tight wavelet frame (Parseval wavelet frame); it is called a Bessel sequence in $H^{s}\left(\mathbb{R}^{d}\right)$ if only the inequality on the right-hand side of (11) holds, where $B$ is called a Bessel bound. Furthermore, we say that $\left(X^{s}\left(\phi ; \varphi_{1}, \varphi_{2}, \ldots, \varphi_{L}\right), X^{-s}\left(\tilde{\phi} ; \tilde{\varphi}_{1}, \tilde{\varphi}_{2}, \ldots, \tilde{\varphi}_{L}\right)\right)$ is a pair of nonhomogeneous dual wavelet frames in $\left(H^{s}\left(\mathbb{R}^{d}\right), H^{-s}\left(\mathbb{R}^{d}\right)\right)$ if the systems $X^{s}\left(\phi ; \varphi_{1}, \varphi_{2}, \ldots, \varphi_{L}\right)$ and $X^{-s}\left(\tilde{\phi} ; \tilde{\varphi}_{1}, \tilde{\varphi}_{2}, \ldots, \tilde{\varphi}_{L}\right)$ are both Bessel sequences in $H^{s}\left(\mathbb{R}^{d}\right)$ and $H^{-s}\left(\mathbb{R}^{d}\right)$, respectively, and

$$
\begin{aligned}
\langle f, g\rangle=\sum_{k \in \mathbb{Z}^{d}}\left\langle f, \tilde{\phi}_{0, k}\right\rangle\left\langle\phi_{0, k}, g\right\rangle & \\
& +\sum_{l=1}^{L} \sum_{j=0}^{\infty} \sum_{k \in \mathbb{Z}^{d}}\left\langle f, \tilde{\varphi}_{l, j, k}^{-s}\right\rangle\left\langle\psi_{l, j, k}^{s}, g\right\rangle
\end{aligned}
$$

holds for all $f \in H^{s}\left(\mathbb{R}^{d}\right)$ and $g \in H^{-s}\left(\mathbb{R}^{d}\right)$. If $\left(X^{s}\left(\phi ; \varphi_{1}, \varphi_{2}, \ldots, \varphi_{L}\right), X^{-s}\left(\tilde{\phi} ; \tilde{\varphi}_{1}, \tilde{\varphi}_{2}, \ldots, \tilde{\varphi}_{L}\right)\right)$ is a pair of dual frames in $\left(H^{s}\left(\mathbb{R}^{d}\right), H^{-s}\left(\mathbb{R}^{d}\right)\right)$, then the reconstruction formulas follow from (12) that

$$
\begin{aligned}
f=\sum_{k \in \mathbb{Z}^{d}}\left\langle f, \tilde{\phi}_{0, k}\right\rangle & \phi_{0, k} \\
& +\sum_{l=1}^{L} \sum_{j=0}^{\infty} \sum_{k \in \mathbb{Z}^{d}}\left\langle f, \tilde{\varphi}_{l, j, k}^{-s}\right\rangle \varphi_{l, j, k}^{s}, f \in H^{s}\left(\mathbb{R}^{d}\right)
\end{aligned}
$$

and

$$
\begin{aligned}
g=\sum_{k \in \mathbb{Z}^{d}}\left\langle g, \phi_{0, k}\right\rangle \tilde{\phi}_{0, k} & \\
& +\sum_{l=1}^{L} \sum_{j=0}^{\infty} \sum_{k \in \mathbb{Z}^{d}}\left\langle g, \varphi_{l, j, k}^{s}\right\rangle \tilde{\varphi}_{l, j, k}^{-s}, g \in H^{-s}\left(\mathbb{R}^{d}\right)
\end{aligned}
$$

with the series converging unconditionally in $H^{s}\left(\mathbb{R}^{d}\right)$ and $H^{-s}\left(\mathbb{R}^{d}\right)$, respectively.

\section{LEMMAS}

In this section, we provide some necessary lemmas which will be used in the proof of the main theorem.

Lemma 1 Let $s \in \mathbb{R}, \phi \in H^{-s}\left(\mathbb{R}^{d}\right)$ and $j \in \mathbb{Z}$. Then, for $f \in H^{s}\left(\mathbb{R}^{d}\right)$ and $k \in \mathbb{Z}^{d}$, the $k$-th Fourier coefficient of $\sum_{l \in \mathbb{Z}^{d}}|\operatorname{det} M|^{j / 2} \hat{f}\left(M^{* j}(\cdot+l)\right) \overline{\hat{\phi}(\cdot+l)}$ is $\left\langle f, \phi_{j, k}\right\rangle$. In particular,

$$
\begin{aligned}
\sum_{l \in \mathbb{Z}^{d}}|\operatorname{det} M|^{j / 2} \hat{f}\left(M^{* j}(\cdot+l)\right) \overline{\hat{\phi}(\cdot+l)} & \\
= & \sum_{k \in \mathbb{Z}^{d}}\left\langle f, \phi_{j, k}\right\rangle \mathrm{e}^{2 \pi i\langle k, \cdot}
\end{aligned}
$$

if $\left\{\phi_{j, k}: k \in \mathbb{Z}^{d}\right\}$ is a Bessel sequence in $H^{-s}\left(\mathbb{R}^{d}\right)$.

Proof: Since $f \in H^{s}\left(\mathbb{R}^{d}\right)$ and $\phi \in H^{-s}\left(\mathbb{R}^{d}\right)$, we have $\hat{f}\left(M^{*^{j}} \cdot \overline{\hat{\phi}(\cdot)} \in L^{1}\left(\mathbb{R}^{d}\right)\right.$, and thus, we can switch the order of summation and integration

$$
\begin{gathered}
\int_{\mathbb{T}^{d}} \sum_{l \in \mathbb{Z}^{d}}|\operatorname{det} M|^{j / 2} \hat{f}\left(M^{*^{j}}(\xi+l) \overline{\hat{\phi}(\xi+l)} \mathrm{e}^{-2 \pi i\langle k, \xi\rangle} \mathrm{d} \xi\right. \\
=|\operatorname{det} M|^{j / 2} \int_{\mathbb{R}^{d}} \hat{f}\left(M^{*^{j}} \xi\right) \overline{\hat{\phi}(\xi)} \mathrm{e}^{-2 \pi i\langle k, \xi\rangle} \mathrm{d} \xi
\end{gathered}
$$


replacing $M^{*^{j}} \xi$ by $\xi$ in (14), we get

$$
\begin{gathered}
\int_{\mathbb{T}^{d}} \sum_{l \in \mathbb{Z}^{d}}|\operatorname{det} M|^{j / 2} \hat{f}\left(M^{*^{j}}(\xi+l) \overline{\hat{\phi}(\xi+l)} \mathrm{e}^{-2 \pi i\langle k, \xi\rangle} \mathrm{d} \xi\right. \\
=|\operatorname{det} M|^{-j / 2} \int_{\mathbb{R}^{d}} \hat{f}(\xi) \overline{\hat{\phi}\left(M^{*^{-j}} \xi\right)} \mathrm{e}^{-2 \pi i\left\langle k, M^{*^{-j}} \xi\right\rangle} \mathrm{d} \xi \\
=\int_{\mathbb{R}^{d}} \hat{f}(\xi) \overline{\left[\phi_{j, k}(\cdot)\right]^{\wedge}(\xi)} \mathrm{d} \xi=\left\langle f, \psi_{j, k}\right\rangle
\end{gathered}
$$

by the Plancherel theorem. So the $k$-th Fourier coefficient of $\sum_{l \in \mathbb{Z}^{d}}|\operatorname{det} M|^{j / 2} \hat{f}\left(M^{* j}(\cdot+l)\right) \overline{\hat{\phi}(\cdot+l)}$ is $\left\langle f, \phi_{j, k}\right\rangle$.

If $\left\{\phi_{j, k}: k \in \mathbb{Z}^{d}\right\}$ is a Bessel sequence in $H^{-s}\left(\mathbb{R}^{d}\right)$, then $\left\{\left\langle f, \phi_{j, k}\right\rangle\right\}_{k \in \mathbb{Z}^{d}} \in \ell^{2}\left(\mathbb{Z}^{d}\right)$, and thus (13) follows directly from (15).

By a careful observation of the proof of Proposition 2.1 in [11], we have the following lemma.

Lemma 2 Let $s \in \mathbb{R}, \phi, \varphi_{1}, \varphi_{2}, \ldots, \varphi_{L} \in H^{s}\left(\mathbb{R}^{d}\right)$. Then $X^{s}\left(\phi ; \varphi_{1}, \varphi_{2}, \ldots, \varphi_{L}\right)$ is a Bessel sequence in $H^{s}\left(\mathbb{R}^{d}\right)$ with bound $B$ if and only if

$$
\begin{aligned}
& \sum_{k \in \mathbb{Z}^{d}}\left|\left\langle g, \phi_{0, k}\right\rangle\right|^{2}+\sum_{l=1}^{L} \sum_{j=0}^{\infty} \sum_{k \in \mathbb{Z}^{d}}\left|\left\langle g, \varphi_{l, j, k}^{s}\right\rangle\right|^{2} \\
& \leqslant B\|g\|_{H^{-s}\left(\mathbb{R}^{d}\right)}^{2} \text { for } \quad g \in H^{-s}\left(\mathbb{R}^{d}\right) .
\end{aligned}
$$

Lemma 3 Let $s \in \mathbb{R}$ and $\phi, \varphi_{1}, \varphi_{2}, \ldots, \varphi_{L} \in H^{s}\left(\mathbb{R}^{d}\right)$. If $X^{s}\left(\phi ; \varphi_{1}, \varphi_{2}, \ldots, \varphi_{L}\right)$ is a Bessel sequence in $H^{s}\left(\mathbb{R}^{d}\right)$ with Bessel bound $B$, then

$|\hat{\phi}(\cdot)|^{2}+\sum_{l=1}^{L} \sum_{j=0}^{\infty} m^{-2 j s}\left|\hat{\varphi}_{l}\left(M^{*^{-j}} \cdot\right)\right|^{2} \leqslant B\left(1+\|\cdot\|^{2}\right)^{-s}$

holds a.e. on $\mathbb{R}^{d}$.

Proof: Since $X^{s}\left(\phi ; \varphi_{1}, \varphi_{2}, \ldots, \varphi_{L}\right)$ is a Bessel sequence in $H^{s}\left(\mathbb{R}^{d}\right)$ with bound $B$, by Lemma 2 , we have

$$
\begin{aligned}
\sum_{k \in \mathbb{Z}^{d}}\left|\left\langle g, \phi_{0, k}\right\rangle\right|^{2}+\sum_{l=1}^{L} \sum_{j=0}^{\infty} \sum_{k \in \mathbb{Z}^{d}}\left|\left\langle g, \varphi_{l, j, k}^{s}\right\rangle\right|^{2} \\
\leqslant B\|g\|_{H^{-s}\left(\mathbb{R}^{d}\right)}^{2} \text { for } \quad g \in H^{-s}\left(\mathbb{R}^{d}\right) .
\end{aligned}
$$

By applying Lemma 1, we compute that

$$
\begin{aligned}
& \sum_{k \in \mathbb{Z}^{d}}\left|\left\langle g, \phi_{0, k}\right\rangle\right|^{2}+\sum_{l=1}^{L} \sum_{j=0}^{\infty} \sum_{k \in \mathbb{Z}^{d}}\left|\left\langle g, \varphi_{l, j, k}^{s}\right\rangle\right|^{2} \\
& =\int_{\mathbb{T}^{d}}\left|\sum_{k \in \mathbb{Z}^{d}} \hat{g}(\xi+k) \overline{\hat{\phi}(\xi+k)}\right|^{2} \mathrm{~d} \xi+\sum_{l=1}^{L} \sum_{j=0}^{\infty} m^{j(d-2 s)} \\
& \times \int_{\mathbb{T}^{d}}\left|\sum_{k \in \mathbb{Z}^{d}} \hat{g}\left(M^{*^{j}}(\xi+k)\right) \overline{\hat{\varphi}_{l}(\xi+k)}\right|^{2} \mathrm{~d} \xi \\
& =\int_{\mathbb{T}^{d}}\left(\sum_{k \in \mathbb{Z}^{d}} \hat{\phi}(\xi+k) \overline{\hat{g}(\xi+k)}\right) \\
& \times\left(\sum_{k \in \mathbb{Z}^{d}} \hat{g}(\xi+k) \overline{\hat{\phi}(\xi+k)}\right) \mathrm{d} \xi+\sum_{l=1}^{L} \sum_{j=0}^{\infty} m^{j(d-2 s)} \\
& \times \int_{\mathbb{T}^{d}}\left(\sum_{k \in \mathbb{Z}^{d}} \hat{\varphi}_{l}(\xi+k) \overline{\hat{g}\left(M^{* j}(\xi+k)\right)}\right) \\
& \times\left(\sum_{k \in \mathbb{Z}^{d}} \hat{g}\left(M^{*^{j}}(\xi+k)\right) \overline{\hat{\varphi}_{l}(\xi+k)}\right) \mathrm{d} \xi \\
& =\int_{\mathbb{R}^{d}} \hat{\phi}(\xi) \overline{\hat{g}(\xi)} \sum_{k \in \mathbb{Z}^{d}} \hat{g}(\xi+k) \overline{\hat{\phi}(\xi+k)} \mathrm{d} \xi \\
& +\sum_{l=1}^{L} \sum_{j=0}^{\infty} m^{-2 j s} \int_{\mathbb{R}^{d}} \hat{\varphi}_{l}\left(M^{*^{-j}} \xi\right) \overline{\hat{g}(\xi)} \\
& \times \sum_{k \in \mathbb{Z}^{d}} \hat{g}\left(\xi+M^{*^{j}} k\right) \overline{\hat{\varphi}_{l}\left(M^{*^{-j}} \xi+k\right)} \mathrm{d} \xi
\end{aligned}
$$

by the Fubini-Tonelli Theorem. Separate the above series into two parts: $k=0$ and $k \neq 0$, we have

$$
\begin{aligned}
& \sum_{k \in \mathbb{Z}^{d}}\left|\left\langle g, \phi_{0, k}\right\rangle\right|^{2}+\sum_{l=1}^{L} \sum_{j=0}^{\infty} \sum_{k \in \mathbb{Z}^{d}}\left|\left\langle g, \varphi_{l, j, k}^{s}\right\rangle\right|^{2} \\
& =\int_{\mathbb{R}^{d}}|\hat{g}(\xi)|^{2}\left[|\hat{\phi}(\xi)|^{2}+\sum_{l=1}^{L} \sum_{j=0}^{\infty} m^{-2 j s}\left|\hat{\varphi}_{l}\left(M^{*^{-j}} \xi\right)\right|^{2}\right] \mathrm{d} \xi \\
& +\int_{\mathbb{R}^{d}} \frac{\hat{g}(\xi)}{0 \neq \neq \in \mathbb{Z}^{d}} \hat{g}(\xi+k)[\hat{\phi}(\xi) \overline{\hat{\phi}(\xi+k)} \\
& \left.\quad+\sum_{l=1}^{L} \sum_{j=0}^{\tau(k)} m^{-2 j s} \hat{\varphi}_{l}\left(M^{*^{-j}} \xi\right) \overline{\hat{\varphi}_{l}\left(M^{*^{-j}}(\xi+k)\right)}\right] \mathrm{d} \xi
\end{aligned}
$$

by the definition of $\tau$. Next, we prove the lemma by contradiction.

Suppose (17) does not hold, then there exists $E \subset$ $\mathbb{R}^{d}$ with $|E|>0$ such that

$$
|\hat{\phi}(\cdot)|^{2}+\sum_{l=1}^{L} \sum_{j=0}^{\infty} m^{-2 j s}\left|\hat{\varphi}_{l}\left(M^{*^{-j}} \cdot\right)\right|^{2}>B\left(1+\|\cdot\|^{2}\right)^{-s}
$$


on $E$, then it follows that

$$
|\hat{\phi}(\cdot)|^{2}+\sum_{l=1}^{L} \sum_{j=0}^{\infty} m^{-2 j s}\left|\hat{\varphi}_{l}\left(M^{*^{-j}} \cdot\right)\right|^{2}>B\left(1+\|\cdot\|^{2}\right)^{-s}
$$

on some $E^{\prime}=E \cap\left([0,1)^{d}+k_{0}\right)$ with $\left|E^{\prime}\right|>0$ and $k_{0} \in$ $\mathbb{Z}^{d}$. Take $g$ such that $\hat{g}(\cdot)=\left(1+\|\cdot\|^{2}\right)^{s / 2} \chi_{E^{\prime}}(\cdot)$ in (20), then we obtain

$$
\|g\|_{H^{-s}\left(\mathbb{R}^{d}\right)}^{2}=\left|E^{\prime}\right|
$$

and

$$
\begin{aligned}
\sum_{k \in \mathbb{Z}^{d}}\left|\left\langle g, \phi_{0, k}\right\rangle\right|^{2}+\sum_{l=1}^{L} \sum_{j=0}^{\infty} \sum_{k \in \mathbb{Z}^{d}}\left|\left\langle g, \varphi_{l, j, k}^{s}\right\rangle\right|^{2} \\
>B\left|E^{\prime}\right|=B\|g\|_{H^{-s}\left(\mathbb{R}^{d}\right)}^{2}
\end{aligned}
$$

contradicting (18). The proof is complete.

\section{NONHOMOGENEOUS DUAL WAVELET FRAMES IN SOBOLEV SPACES}

This section devotes to characterization of the nonhomogeneous dual wavelet frames in $\left(H^{s}\left(\mathbb{R}^{d}\right), H^{-s}\left(\mathbb{R}^{d}\right)\right)$. $\mathrm{T}$ he following theorem provides us with such characterization.

Theorem 1 Given $s \in \mathbb{R}$, let $\phi, \varphi_{1}, \varphi_{2}, \ldots, \varphi_{L} \in H^{s}\left(\mathbb{R}^{d}\right)$ and $\tilde{\phi}, \tilde{\varphi}_{1}, \tilde{\varphi}_{2}, \ldots, \tilde{\varphi}_{L} \in H^{-s}\left(\mathbb{R}^{d}\right)$. Define wavelet systems $X^{s}\left(\phi ; \varphi_{1}, \varphi_{2}, \ldots, \varphi_{L}\right) \quad$ and $X^{-s}\left(\tilde{\phi} ; \tilde{\varphi}_{1}, \tilde{\varphi}_{2}, \ldots, \tilde{\varphi}_{L}\right)$ as in (9) and (10), respectively. Suppose that $X^{s}\left(\phi ; \varphi_{1}, \varphi_{2}, \ldots, \varphi_{L}\right)$ and $X^{-s}\left(\tilde{\phi} ; \tilde{\varphi}_{1}, \tilde{\varphi}_{2}, \ldots, \tilde{\varphi}_{L}\right)$ are both Bessel sequences in $H^{s}\left(\mathbb{R}^{d}\right)$ and $H^{-s}\left(\mathbb{R}^{d}\right)$, respectively. Then $\left(X^{s}\left(\phi ; \varphi_{1}, \varphi_{2}, \ldots, \varphi_{L}\right), X^{-s}\left(\tilde{\phi} ; \tilde{\varphi}_{1}, \tilde{\varphi}_{2}, \ldots, \tilde{\varphi}_{L}\right)\right)$ is a pair of dual frames in $\left(H^{s}\left(\mathbb{R}^{d}\right), H^{-s}\left(\mathbb{R}^{d}\right)\right)$ if and only if, for each $k \in \mathbb{Z}^{d}$,

$$
\hat{\phi}(\cdot) \overline{\hat{\tilde{\phi}}(\cdot+k)}+\sum_{l=1}^{L} \sum_{j=0}^{\tau(k)} \hat{\varphi}_{l}\left(M^{*^{-j}} \cdot\right) \overline{\hat{\tilde{\varphi}}_{l}\left(M^{*^{-j}}(\cdot+k)\right)}=\delta_{0, k}
$$$$
\text { a.e. on } \mathbb{R}^{d} \text {. }
$$

Proof: Since $X^{s}\left(\phi ; \varphi_{1}, \varphi_{2}, \ldots, \varphi_{L}\right)$ and $X^{-s}\left(\tilde{\phi} ; \tilde{\varphi}_{1}\right.$, $\left.\tilde{\varphi}_{2}, \ldots, \tilde{\varphi}_{L}\right)$ are both Bessel sequences in $H^{s}\left(\mathbb{R}^{d}\right)$ and $H^{-s}\left(\mathbb{R}^{d}\right)$, to prove $\left(X^{s}\left(\phi ; \varphi_{1}, \varphi_{2}, \ldots, \varphi_{L}\right)\right.$, $\left.X^{-s}\left(\tilde{\phi} ; \tilde{\varphi}_{1}, \tilde{\varphi}_{2}, \ldots, \tilde{\varphi}_{L}\right)\right)$ is a pair of dual frames for $\left(H^{s}\left(\mathbb{R}^{d}\right), H^{-s}\left(\mathbb{R}^{d}\right)\right)$, it is sufficient to prove that, for $f \in H^{s}\left(\mathbb{R}^{d}\right)$ and $g \in H^{-s}\left(\mathbb{R}^{d}\right)$,

$$
\begin{gathered}
\sum_{k \in \mathbb{Z}^{d}}\left\langle f, \tilde{\phi}_{0, k}\right\rangle\left\langle\phi_{0, k}, g\right\rangle+\sum_{l=1}^{L} \sum_{j=0}^{\infty} \sum_{k \in \mathbb{Z}^{d}}\left\langle f, \tilde{\varphi}_{l, j, k}^{-s}\right\rangle\left\langle\varphi_{l, j, k}^{s}, g\right\rangle \\
=\langle f, g\rangle .
\end{gathered}
$$

By using the Plancherel theorem and Lemma 1, we compute that

$$
\begin{aligned}
& \sum_{k \in \mathbb{Z}^{d}}\left\langle f, \tilde{\phi}_{0, k}\right\rangle\left\langle\phi_{0, k}, g\right\rangle+\sum_{l=1}^{L} \sum_{j=0}^{\infty} \sum_{k \in \mathbb{Z}^{d}}\left\langle f, \tilde{\varphi}_{l, j, k}^{-s}\right\rangle\left\langle\varphi_{l, j, k}^{s}, g\right\rangle \\
& =\int_{\mathbb{T}^{d}}\left(\sum_{k \in \mathbb{Z}^{d}} \hat{f}(\xi+k) \overline{\hat{\tilde{\phi}}(\xi+k)}\right) \times \\
& \left(\sum_{k \in \mathbb{Z}^{d}} \hat{\phi}(\xi+k) \overline{\hat{g}(\xi+k)}\right) \mathrm{d} \xi \\
& +\sum_{l=1}^{L} \sum_{j=0}^{\infty} m^{j} \int_{\mathbb{T}^{d}}\left(\sum_{k \in \mathbb{Z}^{d}} \hat{f}\left(M^{*^{j}}(\xi+k) \overline{\hat{\tilde{\varphi}}_{l}(\xi+k)}\right)\right. \\
& \times\left(\sum_{k \in \mathbb{Z}^{d}} \hat{\varphi}_{l}(\xi+k) \overline{\hat{g}\left(M^{*^{j}}(\xi+k)\right)}\right) \mathrm{d} \xi \\
& =\int_{\mathbb{R}^{d}} \sum_{k \in \mathbb{Z}^{d}} \hat{f}(\xi+k) \overline{\tilde{\tilde{\phi}}(\xi+k)} \hat{\phi}(\xi) \overline{\hat{g}(\xi)} \mathrm{d} \xi \\
& +\sum_{l=1}^{L} \sum_{j=0}^{\infty} m^{j} \int_{\mathbb{R}^{d}} \sum_{k \in \mathbb{Z}^{d}} \hat{f}\left(M^{*^{j}}(\xi+k)\right) \times \\
& \overline{\hat{\varphi}_{l}(\xi+k)} \hat{\varphi}_{l}(\xi) \overline{\hat{g}\left(M^{*^{j}} \xi\right)} \mathrm{d} \xi
\end{aligned}
$$

Separate the above series into two parts: $k=0$ and $k \neq 0$, we get

$$
\begin{aligned}
& \sum_{k \in \mathbb{Z}^{d}}\left\langle f, \tilde{\phi}_{0, k}\right\rangle\left\langle\phi_{0, k}, g\right\rangle+\sum_{l=1}^{L} \sum_{j=0}^{\infty} \sum_{k \in \mathbb{Z}^{d}}\left\langle f, \tilde{\varphi}_{l, j, k}^{-s}\right\rangle\left\langle\varphi_{l, j, k}^{s}, g\right\rangle \\
& =\int_{\mathbb{R}^{d}} \hat{f}(\xi) \overline{\hat{g}(\xi)}[\hat{\phi}(\xi) \overline{\hat{\tilde{\phi}}(\xi)} \\
& \quad+\sum_{l=1}^{L} \sum_{j=0}^{\infty} \hat{\varphi}_{l}\left(M^{*^{-j}} \xi \overline{\hat{\tilde{\varphi}}_{l}\left(M^{*^{-j}} \xi\right)}\right] \mathrm{d} \xi \\
& \quad+\int_{\mathbb{R}^{d}} \overline{\hat{g}(\xi)}\left[\sum_{0 \neq k \in \mathbb{Z}^{d}} \hat{f}(\xi+k) \hat{\phi}(\xi) \overline{\hat{\tilde{\phi}}(\xi+k)}\right. \\
& \left.+\sum_{l=1}^{L} \sum_{j=0}^{\infty} \sum_{0 \neq k \in \mathbb{Z}^{d}} \hat{f}\left(\xi+M^{*^{j}} k\right) \hat{\varphi}_{l}\left(M^{*^{-j}} \xi\right) \overline{\hat{\tilde{\varphi}}_{l}\left(M^{*^{-j}} \xi+k\right)}\right] \mathrm{d} \xi
\end{aligned}
$$

$$
\begin{aligned}
=\int_{\mathbb{R}^{d}} \hat{f}(\xi) \overline{\hat{g}(\xi)}[\hat{\phi}(\xi) \overline{\hat{\tilde{\phi}}(\xi)} \\
\quad+\sum_{l=1}^{L} \sum_{j=0}^{\infty} \hat{\varphi}_{l}\left(M^{*^{-j}} \xi \overline{\hat{\tilde{\varphi}}_{l}\left(M^{*^{-j}} \xi\right)}\right] \mathrm{d} \xi \\
\quad+\int_{\mathbb{R}^{d}} \overline{\hat{g}(\xi)} \sum_{0 \neq k \in \mathbb{Z}^{d}} \hat{f}(\xi+k)[\hat{\phi}(\xi) \overline{\hat{\tilde{\phi}}(\xi+k)} \\
\left.\quad+\sum_{l=1}^{L} \sum_{j=0}^{\tau(k)} \hat{\varphi}_{l}\left(M^{*^{-j}} \xi\right) \overline{\hat{\tilde{\varphi}}_{l}\left(M^{*^{-j}}(\xi+k)\right)}\right] \mathrm{d} \xi .
\end{aligned}
$$


Therefore, (25) can be written as

$$
\begin{aligned}
& \int_{\mathbb{R}^{d}} \hat{f}(\xi) \overline{\hat{g}(\xi)}[\hat{\phi}(\xi) \overline{\hat{\tilde{\phi}}(\xi)} \\
& +\sum_{l=1}^{L} \sum_{j=0}^{\infty} \hat{\varphi}_{l}\left(M^{*^{-j}} \xi \overline{\hat{\tilde{\varphi}}_{l}\left(M^{*^{-j}} \xi\right)}\right] \mathrm{d} \xi \\
& +\int_{\mathbb{R}^{d}} \overline{\hat{g}(\xi)} \sum_{0 \neq k \in \mathbb{Z}^{d}} \hat{f}(\xi+k)[\hat{\phi}(\xi) \overline{\hat{\tilde{\phi}}(\xi+k)} \\
& \left.+\sum_{l=1}^{L} \sum_{j=0}^{\tau(k)} \hat{\varphi}_{l}\left(M^{*^{-j}} \xi\right) \overline{\hat{\tilde{\varphi}}_{l}\left(M^{*^{-j}}(\xi+k)\right)}\right] \mathrm{d} \xi \\
& \quad=\int_{\mathbb{R}^{d}} \hat{f}(\xi) \overline{\hat{g}(\xi)} d \xi .
\end{aligned}
$$

Obviously, (24) implies (28). Now, we need to show that (28) also implies (24) to complete the proof.

Suppose (28) holds. By applying Lemma 3 and the Cauchy-Schwarz inequality, we know the series

$$
\hat{\phi}(\cdot) \overline{\hat{\tilde{\phi}}(\cdot+k)}+\sum_{l=1}^{L} \sum_{j=0}^{\tau(k)} \hat{\varphi}_{l}\left(M^{*^{-j}} \cdot\right) \overline{\hat{\tilde{\varphi}}_{l}\left(M^{*^{-j}}(\cdot+k)\right)}
$$

with $k \in \mathbb{Z}^{d}$ converges absolutely a.e. on $\mathbb{R}^{d}$, and thus belongs to $L^{\infty}\left(\mathbb{R}^{d}\right)$. Therefore, almost every point in $\mathbb{R}^{d}$ is a Lebesgue point. Let $\xi_{0} \in \mathbb{R}^{d}$ be such a point. For $0<\epsilon<1 / 3$, take $f$ and $g$ such that

$$
\hat{f}(\cdot)=\frac{\left(1+\|\cdot\|^{2}\right)^{-s / 2} \chi_{B\left(\xi_{0}, \epsilon\right)}(\cdot)}{\sqrt{\left|B\left(\xi_{0}, \epsilon\right)\right|}}
$$

and

$$
\hat{g}(\cdot)=\frac{\left(1+\|\cdot\|^{2}\right)^{s / 2} \chi_{B\left(\xi_{0}, \epsilon\right)}(\cdot)}{\sqrt{\left|B\left(\xi_{0}, \epsilon\right)\right|}}
$$

in (28), where $B\left(\xi_{0}, \epsilon\right)=\left\{\xi \in \mathbb{R}^{d}:\left|\xi-\xi_{0}\right|<\epsilon\right\}$. Then we have

$$
\begin{aligned}
& \frac{1}{\left|B\left(\xi_{0}, \epsilon\right)\right|} \int_{B\left(\xi_{0}, \epsilon\right)}[\hat{\phi}(\xi) \overline{\hat{\tilde{\phi}}(\xi)} \\
& \quad+\sum_{l=1}^{L} \sum_{j=0}^{\infty} \hat{\varphi}_{l}\left(M^{*^{-j}} \xi \overline{\hat{\tilde{\varphi}}_{l}\left(M^{*^{-j}} \xi\right)}\right] \mathrm{d} \xi=1,
\end{aligned}
$$

and letting $\epsilon \rightarrow 0$, we obtain for $\xi_{0} \in \mathbb{R}^{d}$,

$\hat{\phi}\left(\xi_{0}\right) \overline{\tilde{\hat{\phi}}\left(\xi_{0}\right)}+\sum_{l=1}^{L} \sum_{j=0}^{\infty} \hat{\varphi}_{l}\left(M^{*^{-j}} \xi_{0}\right) \overline{\hat{\tilde{\varphi}}_{l}\left(M^{*^{-j}} \xi_{0}\right)}=1$.

For $0 \neq k_{0} \in \mathbb{Z}^{d}$, take $f$ and $g$ such that

$$
\hat{f}\left(\cdot+k_{0}\right)=\frac{\left(1+\|\cdot\|^{2}\right)^{-s / 2} \chi_{B\left(\xi_{0}, \epsilon\right)}(\cdot)}{\sqrt{\left|B\left(\xi_{0}, \epsilon\right)\right|}}
$$

and

$$
\hat{g}(\cdot)=\frac{\left(1+\|\cdot\|^{2}\right)^{s / 2} \chi_{B\left(\xi_{0}, \epsilon\right)}(\cdot)}{\sqrt{\left|B\left(\xi_{0}, \epsilon\right)\right|}}
$$

in (28), where $0<\epsilon<1 / 3$. Then we have

$$
\begin{aligned}
& \frac{1}{\left|B\left(\xi_{0}, \epsilon\right)\right|} \int_{B\left(\xi_{0}, \epsilon\right)}\left[\hat{\phi}(\xi) \overline{\hat{\tilde{\phi}}\left(\xi+k_{0}\right)}+\right. \\
& \quad \sum_{l=1}^{L} \sum_{j=0}^{\tau\left(k_{0}\right)} \hat{\varphi}_{l}\left(M^{\left.*^{-j} \xi\right)} \overline{\hat{\tilde{\varphi}}_{l}\left(M^{*^{-j}}\left(\xi+k_{0}\right)\right)}\right] \mathrm{d} \xi=0,
\end{aligned}
$$

and letting $\epsilon \rightarrow 0$, we obtain for $\xi_{0} \in \mathbb{R}^{d}$ that

$$
\hat{\phi}\left(\xi_{0}\right) \overline{\hat{\phi}\left(\xi_{0}+k_{0}\right)}+\sum_{l=1}^{L} \frac{\sum_{j=0}^{\tau\left(k_{0}\right)} \hat{\varphi}_{l}\left(M^{*^{-j}} \xi_{0}\right) \times}{\overline{\hat{\varphi}_{l}\left(M^{*^{-j}}\left(\xi_{0}+k_{0}\right)\right)}}=0 .
$$

By the arbitrariness of $\xi_{0}$ and $0 \neq k_{0} \in \mathbb{Z}^{d}$, then we obtain (24). The proof has been completed.

Acknowledgements: This work is supported by the National Natural Science Foundation of China (Grant Nos. 11961072, 62041212), the Natural Science Basic Research Program of Shaanxi (Grant Nos. 2020JM-547, 2020JM-548), and the Doctoral Research Project of Yan'an University (Grant No. YDBK2017-21).

\section{REFERENCES}

1. Cai JF, Dong B, Shen Z (2016) Image restoration: A wavelet frame based model for piecewise smooth functions and beyond. Appl Comput Harmon Anal 41, 94-138.

2. Dong B (2017) Sparse representation on graphs by tight wavelet frames and applications. Appl Comput Harmon Anal 42, 452-479.

3. Stevenson R (2003) Adaptive solution of operator equations using wavelet frames. SIAM J Numer Anal 41, 1074-1100.

4. Han B (1997) On dual wavelet tight frames. Appl Comput Harmon Anal 4, 380-413.

5. Bownik M (2000) A characterization of affine dual frames in $L^{2}\left(\mathbb{R}^{n}\right)$. Appl Comput Harmon Anal 8, 203-221.

6. Christensen O, Kim HO, Kim RY (2016) On extensions of wavelet systems to dual pairs of frames. Adv Comput Math 42, 489-503.

7. Christensen O, Kim HO, Kim RY (2014) On parseval wavelet frames with two or three generators via the unitary extension principle. Canad Math Bull 57, 254-263.

8. Ehler M, Han B (2008) Wavelet bi-frames with few generators from multivariate refinable functions. Appl Comput Harmon Anal 25, 407-414.

9. Ron A, Shen Z (1997) Affine systems in $L^{2}\left(\mathbb{R}^{d}\right)$ : the analysis of the analysis operator. $J$ Funct Anal 148, 408-447.

10. Ron A, Shen Z (1997) Affine systems in $L^{2}\left(\mathbb{R}^{d}\right)$ II: Dual systems. J Fourier Anal Appl 3, 617-637. 
11. Han B, Shen Z (2009) Dual wavelet frames and Riesz bases in Sobolev spaces. Constr Approx 29, 369-406.

12. Han B, Shen Z (2009) Characterization of Sobolev spaces of arbitrary smoothness using nonstationary tight wavelet frames. Isr J Math 172, 371-398.

13. Han B (2017) Homogeneous wavelets and framelets with the refinable structure. Sci China Math 60, 2173-2198.

14. Han B (2012) Nonhomogeneous wavelet systems in high dimensions. Appl Comput Harmon Anal 32, 169-196.
15. Han B (2010) Pairs of frequency-based nonhomogeneous dual wavelet frames in the distribution space. Appl Comput Harmon Anal 29, 330-353.

16. Romero JR, Alexander SK, Baid S, Jain S, Papadakis M (2009) The geometry and the analytic properties of isotropic multiresolution analysis. Adv Comput Math 31, 283-328.

17. Atreas N, Melas A, Stavropoulos T (2014) Affine dual frames and extension principles. Appl Comput Harmon Anal 36, 51-62. 